\title{
Tratamento da depressão bipolar
}

\section{The Treatment of Bipolar Depression}

\author{
BENY LAFER ${ }^{1}$ \\ MARCIA BRITTO DE \\ MACEDO SOARES ${ }^{2}$
}

\begin{abstract}
Resumo
O tratamento da depressão bipolar tem sido tema de debate. O uso de antidepressivos, principalmente tricíclicos, nestes pacientes está associado a piores desfechos clínicos. Estudos apontam para uma eficácia limitada de estabilizadores tradicionais como lítio, valproato e carbamazepina no tratamento da depressão bipolar. Em casos de depressão mais grave, há indicativos de que os antidepressivos podem ser úteis, sendo recomendado o uso concomitante de um estabilizador do humor. Novos agentes como a lamotrigina têm sido propostos como efetivos no tratamento da depressão bipolar. Estudos recentes utilizando lamotrigina sugerem a sua eficácia e seguraça no tratamento da depressão bipolar.
\end{abstract}

Palavras-chave: Depressão bipolar, tratamento, antidepressivos.

\begin{abstract}
The treatment of bipolar depression has been an area of debate. The use of antidepressants, particularly the triciclics, has been associated with worse clinical outcomes. Evidence points to a limited efficacy of traditional mood stabilizers such as lithium, valproate and carbamazepine in the treatment of bipolar depression. In cases where depression is more severe, there is evidence that antidepressants may be useful. The use of antidepressants should be in association with a mood stabilizer. New agents such as lamotrigine have been put forward as effective in the treatment of bipolar depression. Recent studies using lamotrigine suggest its efficacy and safety in the treatment of bipolar depression.
\end{abstract}

Key words: Bipolar depression, treatment, antidepressants.
Recebido: 17/11/2004 - Aceito: 07/01/2005

1 Professor Assistente Doutor do Departamento de Psiquiatria da FMUSP. Coordenador do Projeto de Assistência e Pesquisa em Transtorno Bipolar (PROMAN) do Instituto de Psiquiatria do Hospital das Clínicas da Faculdade de Medicina da Universidade de São Paulo (IPq-HC-FMUSP).

2 Mestre em Medicina pela FMUSP. Médica psiquiatra colaboradora do PROMAN-IPq-HC-FMUSP. Endereço para correspondência: Beny Lafer. Projeto Transtorno Bipolar (PROMAN). Instituto de Psiquiatria, Rua Ovidio Pires de Campos S/N - 05403-010 - São Paulo - SP; e-mail: blafer@usp.br 


\section{Introdução}

Adepressão bipolar, ou seja, episódios depressivos que incidem em portadores do transtorno bipolar (TB), é um dos temas prioritários de pesquisas recentes. Aexistência de diferenças fisiopatológicas e psicopatológicas entre a depressão unipolar e a bipolar foi sugerida a partir de estudos genéticos, evolutivos e clínicos (Goodwin e Jamison, 1990). A apresentação clínica da depressão bipolar também é uma questão controversa, com discussões sobre um possível predomínio de sintomas atípicos (inversão dos sintomas vegetativos e aumento da sensibilidade à rejeição), ou de sintomas melancólicos e de retardo psicomotor. Além disso, a questão relativa ao uso, ou não, de antidepressivos em seu tratamento tem sido objeto freqüente de discussões. Temos, por um lado, relatos sobre o desenvolvimento de episódios hipomaníacos/maníacos (ciclagem) e um possível aumento na freqüência dos ciclos em associação ao seu uso e, por outro, a descrição de um potencial benefício no uso de antidepressivos nos casos mais graves. Neste artigo, discutiremos o diagnóstico e o tratamento (agudo e de continuação) da depressão bipolar, com ênfase no diagnóstico diferencial, no uso de antidepressivos e de novos anticonvulsivantes e antipsicóticos.

\section{Sintomatologia da depressão bipolar versus a depressão unipolar}

A diferenciação entre a depressão unipolar, especialmente a depressão recorrente, e a depressão bipolar pode ser difícil. Eventuais sintomas clínicos sugestivos de que o portador integre o denominado "espectro bipolar" são de difícil detecção, além de sua especificidade ser questionável. O diagnóstico diferencial pode ser particularmente difícil em situações como, por exemplo, episódios depressivos em indivíduos hipertímicos e episódios mistos com predomínio de sintomas depressivos (Koukopoulos e Koukopoulos, 1999; Akiskal e Pinto, 1999; Akiskal et al., 2000).

Kraepelin (apud Goodwin e Jamison, 1990) descreveu que os episódios depressivos integrantes da doença maníaco-depressiva apresentariam uma maior inibição volitiva e um menor grau de disforia do que a melancolia. Ao longo das últimas décadas, características clínicas como início mais precoce, instalação abrupta, maior freqüência de episódios, maior número de episódios, maior freqüência de sintomas psicóticos, incidência no puerpério e maior risco de suicídio foram relacionadas à depressão bipolar (Akiskal et al., 1983; Goodwin e Jamison, 1990).

Mitchell et al. (2001), a partir de um estudo comparativo com controles unipolares, propuseram que a lentificação psicomotora, associada à hipersonia e à presença de sintomas psicóticos, poderia indicar uma "assinatura" da depressão bipolar. Parker et al., em 2000, já haviam descrito uma maior freqüência dos subtipos melancólico e psicótico entre portadores de TB em depressão. Akiskal et al. (1995) e Coryell et al. (1995) sugeriram, inclusive, que a presença de sintomas psicóticos seria um fator preditivo de ciclagem para mania. Benazzi (1999a), contudo, não encontrou diferenças significativas entre unipolares e bipolares manifestando depressões psicóticas.

Outros autores, como Akiskal (1996) e Benazzi (1999b), defendem que a depressão bipolar apresenta mais freqüentemente sintomas atípicos, com inversão dos sintomas vegetativos e maior sensibilidade à rejeição, particularmente em portadores de TB tipo II, o que foi confirmado pelos achados de Benazzi e Rihmer (2000). Robertson et al. (1996), porém, não observaram diferenças no perfil de sintomas atípicos entre deprimidos bipolares e unipolares.

A diferenciação entre episódios depressivos unipolares e bipolares é importante, pois o tratamento da depressão bipolar inclui, necessariamente, o uso de estabilizadores de humor, pelo risco de ciclagem para mania e de possível piora do curso e do prognóstico da doença, como será discutido adiante.

\section{0 tratamento da depressão bipolar}

\section{Estabilizadores do humor}

Com base em artigos originais e de revisão, os dados disponíveis indicam que, entre os estabilizadores do humor clássicos, o lítio apresenta a maior eficácia antidepressiva e é a primeira opção para o tratamento de episódios depressivos leves e moderados em portadores de TB. A carbamazepina e o valproato não apresentam efeitos antidepressivos robustos, de acordo com os resultados até agora relatados (Sachs et al., 2000a; Goodwin, 2003).

\section{Antidepressivos}

\section{Eficácia na fase aguda}

A questão sobre o uso de antidepressivos no tratamento da depressão bipolar tem sido objeto de discussões recentes, mas poucos estudos que incluíram uma população superior a dez indivíduos foram realizados até o momento (Sachs et al., 2000a; Goodwin, 2003; Keck e McElroy, 2003) (Tabela 1).

Não há evidências sobre a maior ou menor eficácia de diferentes compostos antidepressivos no tratamento da depressão bipolar. Atualmente, a escolha por uma ou outra substância reside no risco da associação com episódios hipomaníacos/maníacos. Segundo artigos de revisão e consensos de especialistas, os inibidores seletivos de recaptação de serotonina (ISRSs) e a bupropiona são considerados os antidepressivos de "primeira opção" para o tratamento da depressão bipolar (Sachs et al., 2000a, b; Thase et al., 2003; Yatham et al., 2003; Keck et al., 2003). Os inibidores da monoamina-oxidase (IMAOs) e a 
Tabela 1. Estudos com antidepressivos no tratamento da DB $(n>10)$.

\begin{tabular}{|c|c|c|c|c|c|}
\hline Autores & N & Desenho & $\begin{array}{c}\text { Duração } \\
\text { (semanas) }\end{array}$ & $\begin{array}{c}\text { Outras } \\
\text { medicações }\end{array}$ & Resultados \\
\hline $\begin{array}{l}\text { Kessel e Holt } \\
\text { (1975) }\end{array}$ & $14 \mathrm{BP}$ & $\begin{array}{l}\text { Duplo-cego } \\
\text { imipramina } \\
\text { versus } \\
\text { maprotilina } \\
\end{array}$ & 6 & - & $\begin{array}{c}\text { Resposta: } \\
\text { imipramina: } 66 \% \\
\text { maprotilina: } 40 \%\end{array}$ \\
\hline $\begin{array}{l}\text { Himmelhoch } \\
\text { et al. } \\
\text { (1982) }\end{array}$ & $\begin{array}{l}29 \mathrm{BP} \\
11 \mathrm{PU} \\
190\end{array}$ & $\begin{array}{l}\text { Duplo-cego } \\
\text { tranil } \\
\text { versus } \\
\text { placebo }\end{array}$ & 6 & - & $\begin{array}{c}\text { Resposta: } \\
\text { tranilcipromina: } 91 \% \\
\text { placebo: } 24 \%\end{array}$ \\
\hline $\begin{array}{c}\text { Baumhachl } \\
\text { et al. } \\
\text { (1989) }\end{array}$ & $33 \mathrm{BP}$ & $\begin{array}{c}\text { Duplo-cego } \\
\text { moclobemida } \\
\text { versus } \\
\text { imipramina } \\
\end{array}$ & 4 & Li e BDZ & $\begin{array}{c}\text { Resposta: } \\
\text { moclobemida: } 53 \% \\
\text { imipramina: } 60 \%\end{array}$ \\
\hline $\begin{array}{l}\text { Cohn } \\
\text { et al. } \\
\text { (1989) }\end{array}$ & $89 \mathrm{BP}$ & $\begin{array}{c}\text { Duplo-cego } \\
\text { imipramina } \\
\text { versus } \\
\text { fluoxetina } \\
\text { versus } \\
\text { placebo }\end{array}$ & $\geq 3$ & $\begin{array}{c}\text { Li: } \\
5 \text { imipramina } \\
11 \text { fluoxetina } \\
6 \text { placebo }\end{array}$ & $\begin{array}{c}\text { Resposta: } \\
\text { imipramina: } 57 \% \\
\text { fluoxetina: } 86 \% \\
\text { placebo: } 38 \%\end{array}$ \\
\hline $\begin{array}{l}\text { Thase } \\
\text { et al. } \\
\text { (1992) }\end{array}$ & $16 \mathrm{BP}$ & $\begin{array}{c}\text { Duplo-cego } \\
\text { tranilcipromina } \\
\text { versus } \\
\text { imipramina } \\
\end{array}$ & 6 & - & $\begin{array}{c}\text { Resposta: } \\
\text { tranilcipromina: } 75 \% \\
\text { descontinuação } \\
\text { imipramina: 50\% } \\
\end{array}$ \\
\hline $\begin{array}{l}\text { Sachs } \\
\text { et al. } \\
\text { (1994) }\end{array}$ & $15 \mathrm{BP}$ & $\begin{array}{c}\text { Duplo-cego } \\
\text { bupropiona } \\
\text { versus } \\
\text { desipramina } \\
\end{array}$ & 8 & Li & $\begin{array}{c}\text { Resposta: } \\
\text { bupropiona: } 55 \% \\
\text { desipramina: } 50 \%\end{array}$ \\
\hline $\begin{array}{l}\text { Young } \\
\text { et al. } \\
\text { (2000) }\end{array}$ & $27 \mathrm{BP}$ & $\begin{array}{c}\text { Duplo-cego } \\
\text { paroxetina + } \\
\text { Li ou VP } \\
\text { versus } \\
\text { Li + VP } \\
\end{array}$ & 6 & $\begin{array}{l}\mathrm{Li} \\
\mathrm{VP}\end{array}$ & $\begin{array}{c}\text { Resposta: } \\
\text { paroxetina + } \\
\text { Li ou VP } \\
= \\
\mathrm{Li}+\mathrm{VP}\end{array}$ \\
\hline $\begin{array}{l}\text { Nemeroff } \\
\text { et al. } \\
\text { (2001) }\end{array}$ & $117 \mathrm{BP}$ & $\begin{array}{c}\text { Duplo-cego } \\
\text { paroxetina } \\
\text { versus } \\
\text { imipramina } \\
\text { versus } \\
\text { placebo }\end{array}$ & 10 & $\begin{array}{l}\mathrm{Li} \\
\text { VP } \\
\text { CBZ }\end{array}$ & $\begin{array}{c}\text { Resposta: } \\
\mathrm{Li}>0,8 \mathrm{mmol}: \\
\mathrm{imi}=\text { paroxetina } \\
\mathrm{Li}<\text { ou }=0,8 \mathrm{mmol}: \\
\text { paroxetina }>\text { placebo }\end{array}$ \\
\hline $\begin{array}{l}\text { Silverstone } \\
\text { (2001) }\end{array}$ & $156 \mathrm{BP}$ & $\begin{array}{l}\text { moclobemida } \\
\text { versus } \\
\text { imipramina }\end{array}$ & 8 & $\begin{array}{c}\text { Estabilizadores } \\
\text { do } \\
\text { humor }\end{array}$ & $\begin{aligned} & \text { Resposta: } \\
& \text { moclobemida } \\
&=\text { imipramina }\end{aligned}$ \\
\hline $\begin{array}{l}\text { Vieta } \\
\text { et al. } \\
\text { (2002) }\end{array}$ & $60 \mathrm{BP}$ & $\begin{array}{l}\text { Simples-cego } \\
\text { venlafaxina } \\
\text { versus } \\
\text { paroxetina }\end{array}$ & 6 & $\begin{array}{c}\text { Estabilizadores } \\
\text { do } \\
\text { humor }\end{array}$ & $\begin{array}{l}\text { Resposta: } \\
\text { venlafaxina } \\
=\text { paroxetina }\end{array}$ \\
\hline $\begin{array}{l}\text { Mclntyre } \\
\text { et al. } \\
\text { (2002) }\end{array}$ & $36 \mathrm{BP}$ & $\begin{array}{l}\text { Simples-cego } \\
\text { bupropiona } \\
\text { versus } \\
\text { topiramato }\end{array}$ & 8 & Estabilizadores do humor & $\begin{array}{c}\text { Resposta: } \\
\text { bupropiona: } 59 \% \\
\text { topiramato: } 56 \%\end{array}$ \\
\hline $\begin{array}{l}\text { Tohen } \\
\text { et al. } \\
\text { (2003) }\end{array}$ & $833 \mathrm{BP}$ & $\begin{array}{c}\text { Duplo-cego } \\
\text { olanzapina } \\
\text { versus } \\
\text { olanzapina + } \\
\text { fluoxetina } \\
\text { versus } \\
\text { placebo }\end{array}$ & 8 & - & $\begin{array}{l}\text { Resposta: } \\
\text { olanzapina + } \\
\text { fluoxetina } \\
>\text { olanzapina } \\
\text { > placebo }\end{array}$ \\
\hline
\end{tabular}


venlafaxina figuram como alternativas, principalmente nos casos em que não se observa resposta satisfatória aos ISRSs ou à bupropiona. Os ADTs apresentam elevado risco de ciclagem, e seu uso poderia ser evitado nestes casos (Sachs et al., 2000 a, b; Thase et al., 2003; Yatham et al., 2003; Keck et al., 2003).

\section{Eficácia na fase de continuação e manutenção}

A continuação do antidepressivo após a remissão do episódio agudo é outra questão controversa. Alguns autores sugerem sua continuação por um período de dois a seis meses ressaltando-se que episódios mais graves podem necessitar de uma fase de continuação mais longa (Sachs et al., 2000 b; Henry, 2003). Outros, como Ghaemi et al. (2001), adotam uma postura mais restritiva, sugerindo a continuação por um período de três meses após a remissão. Esta questão polêmica envolve o risco da ciclagem, por um lado, e o de uma recaída depressiva quando da retirada do antidepressivo, por outro.

\section{Mania associada ao uso de antidepressivos}

Existem na literatura vários relatos da ocorrência de sintomas maníacos associados ao tratamento com antidepressivos, e uma descrição destes artigos pode ser encontrada na revisão de Tamada (2002). Indivíduos com um padrão de evolução "depressão-maniaeutimia”, com antecedentes de múltiplas exposições a antidepressivos, história de abuso ou dependência de álcool ou outras substâncias psicoativas e antecedentes de ciclagem, teriam maior risco de apresentar ciclagem associada ao uso de antidepressivos (Koukopoulos et al., 1980; Boerlin et al., 1998; Goldberg et al., 2001, Henry et al., 2001).

A potencial aceleração do ciclo também foi relacionada ao uso de antidepressivos. Altshuler et al. (1995) sugeriram que a mania associada ao antidepressivo poderia ser um marcador de maior vulnerabilidade à aceleração do ciclo induzida pelos antidepressivos. Estudos anteriores já haviam associado a indução de ciclagem rápida à administração contínua ou intermitente de antidepressivos (Koukopoulos et al., 1980; 1983; Wehr et al., 1979; 1988). Os fatores de risco sugeridos para o desenvolvimento de ciclagem rápida, segundo estes estudos, foram: sexo feminino, antecedentes de diversos episódios de mania, antecedentes de ciclagem rápida, temperamento pré-mórbido ciclotímico, diagnóstico de TB tipo I e comorbidade com hipotireoidismo. Deve-se lembrar, contudo, que essas observações foram feitas a partir de estudos abertos e, em sua maioria, retrospectivos. Esses achados ainda precisam ser confirmados por meio de estudos sistematizados e prospectivos. A indução de mania foi também relacionada à suspensão de antidepressivos, mas são necessários estudos mais aprofundados para o estabelecimento de uma relação causal (Ali e Milev, 2003; Benazzi, 2002).
Recentemente, dados publicados por Altshuler et al. (2001) relataram que a interrupção precoce dos antidepressivos (antes de seis meses após a remissão) associou-se a um aumento de cerca de três vezes no risco de uma recaída depressiva, sugerindo que a continuação do antidepressivo por um período mais longo poderia levar à melhor evolução desses pacientes. Esses achados foram confirmados por um estudo prospectivo (Altshuler et al., 2003), no qual a descontinuação precoce dos antidepressivos se associou a um aumento no risco de recaída em quatro vezes, em uma população de portadores de TB tipo I. Wehr et al. (1988), contudo, observaram que a recaída depressiva pode acontecer mesmo na vigência do uso de antidepressivos, especialmente em pacientes com antecedentes de ciclagem associada aos antidepressivos.

Em suma, ainda não existe um consenso em relação à duração do tratamento de continuação com antidepressivos. Essa decisão deve ser individualizada para cada portador, levando-se em conta, por exemplo, o padrão de recorrências, os antecedentes de ciclagem associada ao uso do antidepressivo e a gravidade do episódio.

\section{Novos anticonvulsivantes}

\section{Lamotrigina}

O efeito antidepressivo da lamotrigina foi evidenciado por sete estudos abertos e dois estudos controlados (Calabrese et al., 1999; Frye et al., 2000). Entre os novos anticonvulsivantes, a lamotrigina é o que apresenta maior número de evidências de eficácia antidepressiva, inclusive no tratamento em monoterapia de episódios depressivos em bipolares tipo I (Calabrese et al., 1999), podendo ser a opção para o tratamento de episódios depressivos leves e moderados (Sachs et al., 2000b). No estudo controlado de Calabrese et al. (1999), foram comparados os efeitos de $50 \mathrm{mg} /$ dia e $200 \mathrm{mg} /$ dia de lamotrigina, com placebo, ( $n=192)$, em um estudo duplo-cego, com duração de sete semanas. Observaram-se $51 \%$ de reposta no grupo que recebeu lamotrigina $200 \mathrm{mg} / \mathrm{dia}, 41 \%$ no grupo que recebeu lamotrigina $50 \mathrm{mg} /$ dia e $26 \%$ no grupo que recebeu placebo. A ciclagem para mania foi considerada igual em ambos os grupos $(5,4 \%$ dos pacientes recebendo lamotrigina e 4,6\% com placebo). Frye et al. (2000), também em um estudo controlado, compararam a eficácia antidepressiva de lamotrigina (dose média de $274 \mathrm{mg} /$ dia), gabapentina (dose média de $3.987 \mathrm{mg} / \mathrm{dia})$ e placebo, por seis semanas $(\mathrm{n}=195)$, e descreveram que a lamotrigina foi superior ao placebo na indução da resposta.

\section{Topiramato}

Estudos abertos sobre os efeitos do topiramato no tratamento de episódios de mania, mistos e de depressão do TB sugeriram um possível benefício em casos 
de má resposta aos tratamentos (Hussain et al., 2001; McIntyre et al., 2002). O estudo de McIntyre et al. (2002), inclusive, que comparou o topiramato e a bupropiona, com desenho simples-cego $(n=36)$, não evidenciou diferenças significativas em relação à eficácia nos dois grupos. São necessários, contudo, estudos duploscegos controlados para a melhor avaliação dos benefícios do topiramato no tratamento da depressão bipolar.

\section{Gabapentina}

No estudo já citado de Frye et al. (2000), que comparou a eficácia da lamotrigina (dose média de $274 \mathrm{mg} /$ dia), da gabapentina (dose média de $3.987 \mathrm{mg} /$ dia) e do placebo, por 6 semanas $(n=195)$, a eficácia antidepressiva da gabapentina foi considerada comparável à do placebo.

Concluindo, entre os novos anticonvulsivantes as evidências obtidas até o momento indicam que a lamotrigina é uma alternativa importante para o tratamento da depressão bipolar, em monoterapia ou em associação. O topiramato pode ser útil, em associação, em casos refratários, e a gabapentina não apresenta efeitos antidepressivos robustos.

\section{Antipsicóticos atípicos}

\section{Olanzapina}

Recentemente os efeitos antidepressivos da olanzapina foram avaliados em um estudo multicêntrico duplo-cego, que incluiu um total de 833 pacientes (Tohen et al., 2003), divididos em três grupos: placebo $(\mathrm{n}=377)$, olanzapina $(\mathrm{n}=370)$ e olanzapina + fluoxetina $(n=86)$. Após oito semanas de tratamento, observou-se remissão em 48,8\% do grupo tratado com olanzapina + fluoxetina, em $32,8 \%$ do grupo tratado com a olanzapina e em $24,5 \%$ do grupo que recebeu placebo. Embora os resultados sejam promissores, futuros estudos são necessários para a melhor determinação do papel da olanzapina em monoterapia no tratamento da depressão bipolar e qual a sua eficácia na associação com outros antidepressivos, além da fluoxetina.

\section{Conclusões}

Os estabilizadores do humor constituem a base do tratamento do portador de TB, não só no tratamento profilático, como também nas fases agudas de depressão e mania. O uso, ou não, de antidepressivos no tratamento dos episódios agudos e, mais ainda, no tratamento de continuação é ainda uma questão polêmica. De forma geral, preconiza-se que depressões bipolares leves e moderadas devem ser tratadas com estabilizadores do humor, especialmente lítio e lamotrigina. Em casos graves, deve-se optar pela introdução de antidepressivos ou eletroconvulsoterapia (ECT). Se houver presença de sintomas psicóticos, deve-se associar um antipsicótico, de preferência atípico, ou fazer a opção pela ECT. Os antidepressivos devem ser sempre usados em associação com estabilizadores do humor, e os mais indicados para o tratamento da depressão são a bupropiona e, entre os ISRSs, a paroxetina. Nos casos mais graves os IMAOs podem ser utilizados, constituindo-se importante alternativa terapêutica.

Tabela 2. Eficácia comparada dos antidepressivos no tratamento da depressão bipolar.

$\begin{array}{cc}\text { Eficácia superior ao placebo } & \text { Eficácia comparável a ADTs } \\ \text { Imipramina: } & \text { Maprotilina: } \\ \text { Fieve et al. (1968); } & \text { Kessel; Holt (1975) } \\ \text { Worral (1979) } & \\ \text { Bupropiona: } & \text { Moclobemida: } \\ \text { Meridith (1983); } & \text { Baumhachl (1989) } \\ \text { Fabre (1983) } & \text { Silverstone (2001) } \\ \text { Paroxetina: } & \text { Bupropiona: } \\ \text { Nemeroff et al. (2001) } & \text { Sachs et al. (1994) } \\ \text { Olanzapina + Fluoxetina: } & \text { Paroxetina: } \\ \text { Tohen et al. (2003) } & \text { Nemeroff et al. (2001) }\end{array}$

Eficácia superior à imipramina

Maprotilina:

Moclobemida:

Baumhachl (1989)

Silverstone (2001)

Bupropiona:

Nemeroff et al. (2001)

\author{
Tranilcipromina: \\ Himmelhoch et al. (1991) \\ Fluoxetina: \\ Cohn et al. (1989)
}


Referências bilbiográficas

Akiskal, H.S.; Walker, P.; PuzAntian, V.R. et al. - Bipolar Outcome in the Course of Depressive Illness: Phenomenologic, Familial and Pharmachologic Predictors. J Affect Disord 5: 115-128, 1983.

AKISKAL, H.S.; MASER, J.D.; ZeLLER, P.J. et al. - Switching from Unipolar to Bipolar II. Na 11-year Prospective Study of Clinical and Temperamental Predictors in 559 Patients. Arch Gen Psychiatry 52: 114-123, 1995.

AKISKAL, H.S. - The Prevalent Clinical Spectrum of Bipolar Disorders: Beyond DSM-IV. J Clin Psychopharmacol 16(Suppl 1): 4S-14S, 1996.

AkISKAL, H.S.; PINTo, O. - The Evolving Bipolar Spectrum: Prototypes I, II, III, IV. Psychiat Clin North Am 22: 517 534, 1999.

AKISKAL, H.S.; BourgeoIs, M.L.; AngSt, J. et al. - Re-evaluating the Prevalence and Diagnostic Composition within the Broad Clinical Spectrum of Bipolar Disorders. J Affect Disord 59: S5-S30, 2000.

Alı, S.; Mılev, R. - Switch to Mania upon Discontinuation of Antidepressants in Patients with Mood Disorders: a Review of Literature. Can J Psychiatry 48: 258-264, 2003.

Altshuler, L.L.; Post, R.M.; Leverich, G.S. et al. - L. Antidepressant-Induced Mania and Cycle Acceleration: A controversy Revisited. Am J Psychiatry 152(8): 1130-1138, 1995.

Altshuler, L.; Kiriakos, L.; Calcagno, J. et al. - The Impact of Antidepressant Discontinuation versus Antidepressant Continuation on 1-Year Risk for Relapse of Bipolar Depression: a Retrospective Chhart Review. J Clin Psychiatry 62: 512-616, 2001.

Altshuler, L.; Suppes, T.; Black, D. et al. - Impact of Antidepressant Discontinuation After Acute Bipolar Depression Remission on Rates of Depressive Relapse at 1-year follow-up. Am J Psychiatry 160: 1252-1262, 2003.

Benazzl, F. - Bipolar versus Unipolar Psychotic Outpatient Depression. J Affect Disord 55: 63-66, 1999a.

BenazzI, F. - Prevalence of Bipolar II Disorder in Atypical Depression. Eur Arch Psychiatry Clin Neurosci 249: 6265, 1999b.

BenazzI, F. - Psychotic Mania in Bipolar II Depression Related to Sertraline Discontinuation. Can J Psychiatry 476: 584-585, 2002.

BenAZZI, F; RIHMER, Z. - Sensitivity and Specificity of DSM-IV Atypical Features for Bipolar II Diagnosis. Psychiatry Res 93: 257-262, 2000.

Boerlin, H.L.; Gituin, M.J.; Zoellner, L.A. et al. - Bipolar Depression and Antidepressant-Induced Mania: a Naturalistic Study. J Clin Psychiatry59(7): 374-379, 1998.

Calabrese, J.R.; Bowden, C.L.; Sachs, G.S. et al. - A DoubleBlind Placebo Controlled Study of Lamotrigine Monotherapy in Outpatients with Bipolar I Depression. J Clin Psychiatry 60: 79-88, 1999.

CoryelL, W.; Endicott, J.; MASE, J.D. et al. - Long-Term Stability of Polarity Distictions in the Affective Disorders. Am J Psychiatry 152: 385-390, 1995.
Frye, M.A.; Ketter, T.A.; Kimbrell, T.A. et al. - A PlaceboControlled Study of Lamotrigine and Gabapentin Monotherapy in Refractory Mood Disorders. J Clin Psychopharmacol 20: 607-614, 2000.

Ghaemi, S.N.; Ko, J.Y.; GoodWIn, F.K. - The Bipolar Spectrum and the Antidepressant View of the World. $J$ Clinical Practice 7: 287-297, 2001.

Goldberg, J.F.; Rabin, A.; Whiteside, J.E. - Prevalence and Risks Associated with Antidepressant-induced Mania. Fourth International Conference on Bipolar Disorder. Pittsburgh, Pennsylvania. Abstract 51. Pittsburgh, 2001.

Goodwin, F.K.; Jamison, K.R. - Manic-depressive Illness. Oxford University Press, New York, 1990.

Goodwin, G.M. - Consensus Group of the British Association of Psychopharmacology. Evidence-Based Guidelines for Treating Bipolar Disorder: Recommendations from the British Association for Psychopharmacology. $J$ Psychopharmacol 17: 149-173, 2003.

Henry, C.; Sorbara, F.; Lacoste, J. et al. - AntidepressantInduced Mania in Bipolar Patients: Identification of Risk Factors. J Clin Psychiatry 62: 249-255, 2001.

Henry, C.; Demotes-Mainard J. - Avoiding Drug-induced Switching in Patients with Bipolar Depression. Drug Saf 6: 337-351, 2003.

Hussain, M.Z.; Chaudhry, Z.A.; Hussain, S. - Topiramate in Treatment of Refractory Bipolar Depression. Bipolar Disord 3: 47, 2001.

Keck Jr., P.E.; McElroY, S.L. - New Approaches in Managing Bipolar Depression. J Clin Psychiatry 64(Suppl 1): 1318, 2003.

Keck Jr., P.E.; Nelson, E.B.; McElroy, S.L. - Advances in the Pharmacologic Tratment of Bipolar Depression. Biol Psychiatry 53: 633-644, 2003.

Koukopoulos, A.; Reginaldi, D.; Laddomada, P. et al. -Course of the Manic-Depressive Cycle and Changes Caused by Treatments. Pharmakopsychiatr Neuropsychopharmakol 13: 156-167, 1980.

Koukopoulos, A.; Caliari, B.; Tundo, A. et al. - Rapid Cyclers, Temperament and Antidepressants. Compr Psychiatry 24: 249-258, 1983.

Koukopoulos, A.; Koukopoulos, A. - Agitated Depression as a Mixed State and the Problem of Melancholia. Psychiat Clin North Am 22: 547-563, 1999.

McIntyre, R.S.; Mancinı, D.A.; McCAnn, S. et al. - Topiramate versus Bupropion SR When Added to Mood Stabilizer Therapy for the Depressive Phase of Bipolar Disorder: a Preliminary Single-blind Study. Bipolar Disord 4: 207213, 2002.

Mitchell, P.B.; Wilhelm, K.; Parker, G. et al. - The Clinical Features of Bipolar Depression: a Comparison with Matched Major Depressive Disorder Patients. J Clin Psychiatry 62: 212-216, 2001.

ParkeR, G.; Roy, K.; Wilhelm, K. et al. - The Nature of Bipolar Depression: Implications for the Definition of $\mathrm{Me}$ lancholia. J Affect Disord 59: 217-224, 2000. 
Robertson, H.A.; Lam, R.W.; Stewart, J.N. et al. - Atypical Depressive Symptoms and Clusters in Unipolar and Bipolar Depression. Acta Psychiatr Scand 94: 421-427, 1996.

Sachs, G.S.; Koslow, C.L.; Ghaemi, S.N. - The Treatment of Bipolar Depression. Bipolar Disord 2: 256-260, 2000a.

Sachs, G.S.; Printz D.J.; Kahn, D.A. et al. - The Expert Consensus Guideline Series: Medication Treatment of Bipolar Disorder. Postgrad Med 1-104, 2000b.

TAMAdA, R.S. - Estudo Comparativo Entre Mania Espontânea e Mania Induzida por Antidepressivo no Transtorno Bipolar: Características Clínicas, Resposta ao Tratamento e Evolução. São Paulo, 2002. Dissertação (Mestrado) - Faculdade de Medicina, Universidade de São Paulo.

Thase, M.E.; Bhargava, M.; Sachs, G.S. - Treatment of Bipolar Depression: Current Status, Continued Challenges and the STEP-BD Approach. Psychiat Clin North Am 26: 495518, 2003.

Tohen, M.; Vieta, E.; Calabrese, J. et al. - Efficacy of Olanzapine and Olanzapine-fluoxetine Combination in the Treatment of Bipolar I Depression. Arch Gen Psychiatry 60: 1079-1088, 2003.

WeHR, T.A.; GoodWIN, F.K. - Rapid Cycling in Manic-depressive Induced by Tryciclic Antidepressants. Arch Gen Psychiatry 36: 555-559, 1979.

WeHR, T.A.; SACK, D.A.; Rosenthal, N.E. etal. - Rapid Cycling Affective Disorder: Contributing Factors and Treatment Responses in 51 Patients. Am J Psychiatry 145: 179-184, 1988.

Yatham, L.N.; Calabrese, J.R.; Kusumakar, V. - Bipolar Depression: Criteria for Tretament Selection, Definition of Refractoriness and Treatment Options. Bipolar Disord 5: 85-97, 2003. 